\title{
Cognitive Capacity Limits Are Remediated by Practice-Induced Plasticity between the Putamen and Pre-Supplementary Motor Area
}

\author{
즈.G. Garner, ${ }^{1,2,3,4}$ M.I. Garrido, ${ }^{1,3,5,6, *}$ and P.E. Dux ${ }^{2, *}$
}

https://doi.org/10.1523/ENEURO.0139-20.2020

\begin{abstract}
${ }^{1}$ Queensland Brain Institute, University of Queensland, Brisbane, Australia, ${ }^{2}$ School of Psychology, University of Queensland, Brisbane, Australia, ${ }^{3}$ Australian Research Council Centre of Excellence for Integrative Brain Function, Australia, ${ }^{4}$ Department of Psychology, University of Birmingham, Birmingham, United Kingdom, ${ }^{5}$ Melbourne School of Psychological Sciences, University of Melbourne, Melbourne, Australia, and ${ }^{6}$ Centre for Advanced Imaging, University of Queensland, Brisbane, Australia
\end{abstract}

\begin{abstract}
Humans show striking limitations in information processing when multitasking yet can modify these limits with practice. Such limitations have been linked to a frontal-parietal network, but recent models of decision-making implicate a striatal-cortical network. We adjudicated these accounts by investigating the circuitry underpinning multitasking in 100 human individuals and the plasticity caused by practice. We observed that multitasking costs, and their practice-induced remediation, are best explained by modulations in information transfer between the striatum and the cortical areas that represent stimulus-response mappings. Specifically, our results support the view that multitasking stems at least in part from taxation in information sharing between the putamen and pre-supplementary motor area (pre-SMA). Moreover, we propose that modulations to information transfer between these two regions leads to practice-induced improvements in multitasking.
\end{abstract}

Key words: cognitive capacity; DCM; multiple demand network; multitasking; practice; subcortical

\section{Significance Statement}

Humans show striking limitations in information processing when multitasking, yet can modify these limits with practice. Such limitations have been linked to a frontal-parietal network, but recent models of decisionmaking implicate a striatal-cortical network. We adjudicated these accounts by investigating the circuitry underpinning multitasking in 100 individuals and the plasticity caused by practice. Our results support the view that multitasking stems at least in part from taxation in information sharing between the putamen and pre-supplementary motor area (pre-SMA). We therefore show that models of cognitive capacity limits must consider how subcortical and cortical structures interface to produce cognitive behaviors, and we propose a novel neurophysiological substrate of multitasking limitations.

\section{Introduction}

Although human information processing is fundamentally limited, the points at which task difficulty or complexity incurs performance costs are malleable with practice. For example, practicing component tasks reduces the response slowing that is typically induced as a consequence of

Received April 8, 2020; accepted June 3, 2020; First published August 17, 2020.

The authors declare no competing financial interests. attempting to complete the same tasks concurrently (multitasking; Telford, 1931; Ruthruff et al., 2001; Strobach and Torsten, 2017). These limitations are currently attributed to competition for representation in a frontal-parietal network (Watanabe and Funahashi, 2014, 2018; Garner and Dux, 2015; Marti et al., 2015), in which the constituent neurons adapt response properties to represent the contents of the

Author contributions: K.G.G., M.I.G., and P.E.D. designed research; K.G.G. performed research; K.G.G. analyzed data; K.G.G., M.I.G., and P.E.D. wrote the paper. 
current cognitive episode (Duncan, 2010, 2013; Woolgar et al., 2011). Despite recent advances, our understanding of the network dynamics that drive multitasking costs and the influence of practice remains unknown. Furthermore, although recent work has focused on understanding cortical contributions to multitasking limitations, multiple theoretical models implicate striatal-cortical circuits as important neurophysiological substrates for the execution of single sensorimotor decisions (Joel et al., 2002; Bornstein and Daw, 2011; Caballero et al., 2018), the formation of stimulus-response representations in frontal-parietal cortex (Ashby et al., 2010; Hélie et al., 2015), and performance of both effortful and habitual sensorimotor tasks (Yin and Knowlton, 2006; Graybiel and Grafton, 2015; Jahanshahi et al., 2015). This suggests that a complete account of cognitive limitations and their practice-induced attenuation also requires interrogation into the contribution of striatal-cortical circuits. We seek to address these gaps in understanding by investigating how multitasking and practice influence network dynamics between striatal and cortical regions previously implicated in the cognitive limitations that give rise to multitasking costs (Garner and Dux, 2015).

We previously observed that improvements in the decodability of component tasks in two regions of the frontal-parietal network, pre-supplementary motor area (preSMA/SMA), the intraparietal sulcus (IPS), and one region of the striatum (putamen) predicted practice-induced multitasking improvements (Garner and Dux, 2015). This implies that practice may not divert performance from the frontal-parietal system, as had been previously assumed (Petersen et al., 1998; Kelly and Garavan, 2005; Yin and Knowlton, 2006; Chein and Schneider, 2012) but, rather, may alleviate multitasking costs by reducing competition for representation within the same system. Moreover, our finding that the putamen showed changes to task decodability that predicted behavioral improvements comparable to what was observed for pre-SMA and IPS implies that rather than stemming from overload of an entirely cortical network (Marois and Ivanoff, 2005; Dux et al., 2006; Marti et al., 2015), multitasking costs are manifest by limitations within a distributed striatal-cortical system. This raises the question of how interactions between

This work was funded by the Australian Research Council Future Fellowship FT120100033 (to P.E.D.), The University of Queensland Foundation Research Excellence Award (P.E.D.), the Australian Research Council-Special Research Initiative (ARC-SRI) Science of Learning Research Centre Grant SR120300015 (to P.E.D.), the Australian Research Council Centre of Excellence for Integrative Brain Function Grant CE140100007 (to M.I.G.), the University of Queensland Fellowship 2016000071 (to M.I.G.), and by a UQ Centennial Scholarship, an International Postgraduate Research Scholarship, and a Marie Sklodowska-Curie Global Fellowship (K.G.G.).

${ }^{*}$ M.I.G. and P.E.D. share senior authorship.

Acknowledgements: We thank the participants for their time; Aiman AlNajjar, Anita Burns, Luke Hearne, and Amy Taylor for assisting with data collection; and David Lloyd for assistance with results visualization.

Correspondence should be addressed to K.G. Garner at getkellygarner@ gmail.com.

https://doi.org/10.1523/ENEURO.0139-20.2020

Copyright (C) 2020 Garner et al.

This is an open-access article distributed under the terms of the Creative Commons Attribution 4.0 International license, which permits unrestricted use, distribution and reproduction in any medium provided that the original work is properly attributed. these brain regions give rise to multitasking costs and how can these be mitigated with practice: do multitasking costs reflect over-taxation of striatal-cortical circuits? Or are they a consequence of competition for representation between cortical areas? Alternately, do multitasking costs stem from limitations in both striatal-cortical and corticocortical connections? Does practice alleviate multitasking costs via modulating all the interregional couplings that give rise to multitasking behavior, or by selectively increasing or reducing couplings between specific regions?

Our aim was to arbitrate between these possibilities by applying dynamic causal modeling (DCM; Friston et al., 2003) to a functional magnetic resonance imaging (fMRI) dataset $(N=100)$ collected while participants performed a multitasking paradigm before and after practice on the same paradigm $(N=50)$ or on an active control task $(N=50$; Garner and Dux, 2015). We sought to first characterize the modulatory influence of multitasking on the network dynamics between the pre-SMA, IPS and putamen, and then to understand how practice modulated these network dynamics in concert with multitasking performance improvements.

\section{Materials and Methods}

\section{Participants}

The MRI scans of the participants $(N=100)$ previously analyzed in Garner and Dux (2015) were included in the present analysis, apart from the data of two participants, for whom some of the scans were corrupted because of experimenter error. The remaining 98 participants had been pseudorandomly allocated to the practice group $[N=48$, mean age: 24.33 (SD: 6.31), 44 F, 44 righthanded] or the control group [ $N=50$, mean age: 24.58 (SD: 5.48), $46 \mathrm{~F}, 45$ right-handed]. All participants received 10 Australian dollars (AUD) per hour for participation. Participants also earned bonus dollars across the three training sessions. Bonus dollars were accrued for high accuracy and for beating RT deadlines ( 20 AUD per participant). For details of the original data point exclusions, we refer the reader to Garner and Dux (2015). The University of Queensland Human Research Ethics Committee approved the study as being within the guidelines of the National Statement on Ethical Conduct in Human Research, and all participants gave informed, written consent.

\section{Experimental protocols}

Participants attended six experimental sessions: a familiarization session, two MRI sessions and three behavioral practice sessions. Familiarization sessions were conducted the Friday before the week of participation, where participants learned the stimulus-response mappings and completed two short runs of the task. The MRI sessions were conducted to obtain pre-practice (Monday session) and post-practice (Friday session) measures. These sessions were held at the same time of day for each participant. Between the two MRI sessions, participants completed three behavioral practice sessions, where they either practiced the multitasking paradigm 
(practice group) or the visual-search task (control group). Participants typically completed one practice session per day, although on occasion, two practice sessions were held on the same day to accommodate participants' schedules (when this occurred, the two sessions were administered with a minimum of 1-h break between them). Participants also completed an online battery of questionnaires that formed part of a different study.

\section{Behavioral tasks}

All tasks were programmed using MATLAB R2010a (MathWorks) and the Psychophysics Toolbox v3.0.9 extension (23). The familiarization and behavioral training sessions were conducted with a 21-inch, Sony Trinitron CRT monitor and a Macintosh 2.5-GHz Minicomputer.

\section{Multitasking paradigm}

For each trial of the multitasking paradigm, participants performed either one (single-task condition) or two (multitask condition) sensorimotor tasks. Both involved a twoalternative discrimination (2-AD), mapping the two stimuli to two responses. For one task, participants were presented with one of two white shapes that were distinguishable in terms of their smooth or spikey texture, presented on a black screen and subtending $\sim 6^{\circ}$ of visual angle. The shapes were created using digital sculpting software (Scluptris Alpha 6) and Photoshop CS6. Participants were required to make the appropriate manual button press to the presented shape, using either the index or middle finger of either the left or right hand (task/hand assignment was counterbalanced across participants). For the other task, participants responded to one of two complex tones using the index or middle finger of the hand that was not assigned to the shape task. The sounds were selected to be easily discriminable from one another. Across both the single-task and multitask trial types, stimuli were presented for $200 \mathrm{~ms}$ and, on multitask trials, were presented simultaneously.

\section{Familiarization session}

During the familiarization session, participants completed two runs of the experimental task. Task runs consisted of 18 trials, divided equally between the three trial types (shape single-task, sound single-task, and multitask trials). The order of trial type presentation was pseudorandomized. The first run had a short intertrial interval (ITI), and the trial structure was as follows: an alerting fixation dot, subtending $0.5^{\circ}$ of visual angle, was presented for $400 \mathrm{~ms}$, followed by the stimulus/stimuli that was presented for $200 \mathrm{~ms}$. Subsequently a smaller fixation dot, subtending $0.25^{\circ}$ of visual angle, was presented for $1800 \mathrm{~ms}$, during which participants were required to respond. Participants were instructed to respond as accurately and quickly as possible to all tasks. For the familiarization session only, performance feedback was then presented until the participant hit the spacebar to continue the task. For example, if the participant had completed the shape task correctly, they were presented with the message "You got the shape task right." If they performed the task incorrectly, the message "Oh no! You got the shape task wrong" was displayed. On multitask trials; feedback was presented for both tasks. If participants failed to achieve at least $5 / 6$ trials correct for each trial type they repeated the run until this level of accuracy was attained.

The second run familiarized participants with the timing of the paradigm to be used during the MRI sessions; a slow event-related design with a long ITI. The alerting fixation was presented for $2000 \mathrm{~ms}$, followed by the $200-\mathrm{ms}$ stimulus presentation, 1800-ms response period, and feedback. Subsequently an ITI, during which the smaller fixation dot remained on screen, was presented for $12,000 \mathrm{~ms}$.

\section{MRI sessions}

Participants completed six long ITI runs in the scanner, with 18 trials per run (six of each trial type, pseudo-randomly ordered for each run), for a total of 108 trials for the session. Trial presentation was identical to the long ITI run presented at the familiarization session, except that feedback was not presented at the end of each trial.

\section{Practice sessions}

All participants were informed that they were participating in a study examining how practice improves attention, with the intention that both the practice and control groups would expect their practice regimen to improve performance. The first practice session began with an overview of the goals of the practice regimen; participants were informed that they were required to decrease their response time (RT), while maintaining a high level of accuracy. The second and third sessions began with visual feedback in the form of a line graph, plotting RT performance from the previous practice sessions.

For each session, participants completed 56 blocks of 18 trials, for a total of 1008 trials, resulting in 3024 practice trials overall. To ensure that participants retained familiarity with the timings of the task as presented in the scanner, between two and four of the blocks in each session used long ITI timings.

The practice group performed the multitasking paradigm, as described above (Familiarization session), except that performance feedback was not displayed after each trial. Over the course of practice, participants from this group performed 1008 trials of each trial type (shape single-task, sound single-task, multitask). Participants in the control group went through the identical procedures to the practice group, except that they completed a visual search task instead of the multitasking paradigm. Participants searched for a "T" target among 7, 11, or 15 rotated "Ls" (to either $90^{\circ}$ or $270^{\circ}$ ). Participants indicated whether the target was oriented to $90^{\circ}$ or $270^{\circ}$, using the first two fingers of their left or right hand (depending on handedness). Over the course of the three practice sessions, participants completed 1008 trials for each set size.

For both groups, performance feedback showed mean RT (collapsed across the two single-tasks for the practice group, and over the three set-sizes for the control group), 
and accuracy, for the previous eight blocks, total points scored, and the RT target for the subsequent eight blocks. If participants met their RT target for over $90 \%$ of trials, and achieved $>90 \%$ accuracy, a new RT target was calculated by taking the $75^{\text {th }}$ percentile of RTs recorded over the previous eight blocks. Furthermore, two points were awarded. If participants did not beat their RT target for over $90 \%$ trials, but did maintain $>90 \%$ accuracy, one point was awarded.

\section{MRI data acquisition}

Images were acquired using a $3 \mathrm{~T}$ Siemens Trio MRI scanner housed at the Centre for Advanced Imaging at The University of Queensland. Participants lay supine in the scanner and viewed the visual display via rear projection onto a mirror mounted on a 12-channel head coil. A T1-weighted anatomic image was collected after the fourth experimental run of the scanning session [repetition time $(T R)=1.9 \mathrm{~s}$, echo time $(T E)=2.32 \mathrm{~ms}$, flip angle $(F A)=9^{\circ}$, field of view $(F O V)=192 \times 230 \times 256 \mathrm{~mm}$, resolution $=1 \mathrm{~mm}^{3}$ ]. Functional T2*-weighted images were acquired parallel to the anterior commissure-posterior commissure plane using a GRE EPI sequence ( $T R=2$ $\mathrm{s}, \mathrm{TE}=35 \mathrm{~ms}, \mathrm{FA}=79^{\circ}, \mathrm{FOV}=192 \times 192 \mathrm{~mm}$, matrix $=$ $64 \times 64$, in-plane resolution $=3 \times 3 \mathrm{~mm}$ ). Each volume consisted of 29 slices (thickness $=3 \mathrm{~mm}$, interslice gap $=0.5 \mathrm{~mm}$ ), providing whole brain coverage. We synchronized the stimulus presentation with the acquisition of functional volumes.

\section{MRI data analysis}

fMRI data were preprocessed using the SPM12 software package (Wellcome Trust Centre for Neuroimaging, London, United Kingdom; http://www.fil.ion.ucl.ac.uk/ spm). Scans from each subject were corrected for slice timing differences using the middle scan as a reference, realigned using the middle scan as a reference, co-registered to the T1 image, spatially normalized into $\mathrm{MNI}$ standard space, and smoothed with a Gaussian kernel of 8-mm full-width at half maximum.

\section{DCM}

To assess the causal direction of information flow between brain regions, we applied DCM, which maps experimental inputs to the observed fMRI output, via hypothesized modulations to neuronal states that are characterized using a biophysically informed generative model (Friston et al., 2003). Parameter estimates are expressed as rate constants (i.e., the rate of change of gross neural activity in one region, given the activity in the coupled brain region) and are fit using Bayesian parameter estimation. It is important to note that any interpretations regarding information transfer between brain regions is based on the assumption that these rate parameters reflect a causal relationship between the regions of interest (ROIs) that is meaningful with regards to task performance. Moreover, with DCM, we seek to model coupling changes between ROls that have been defined a priori and that the presence of such interregional couplings are postulated. It is possible that any observed coupling changes could be driven by a third node that is not included in the proposed network architecture. Moreover, the currently proposed architectures certainly do not reflect the entire network that underpins multitasking of sensorimotor tasks.

\section{DCM implementation}

Implementation of DCM requires definition of endogenous connections within the network (A parameters), the modulatory influence of experimental factors (B parameters), and the influence of exogenous/driving inputs into the system (e.g., sensory inputs, C parameters; Friston et al., 2003). We implemented separate DCMs to investigate (1) the modulatory influence of multitasking on the prepractice data, and (2) the modulatory influence of practice on the pre-practice to post-practice data.

To make inferences regarding the modulatory influence of multitasking, we defined our endogenous network as comprising reciprocal connectivity between all three of our ROIs, on the basis of anatomic and functional evidence for connections between all three of them (Alexander et al., 1986; Cavada and Goldman-Rakic, 1989; Luppino et al., 1993; Wise et al., 1997; Haber, 2016). To address our theoretically motivated question regarding the locus of modulatory influence of the multitasking, we first implemented all 63 possible combinations of the modulatory influence of the multitasking (i.e., allowing each combination of connections to be modulated by the multitasking factor; for an illustration of the model architectures, see Extended Data Fig. 2-2) and then grouped the model space into three families: those that allowed any combination of corticocortical modulations, but not striatal-cortical (corticocortical family, with three models in total $M_{1-3}=3$ ), those that allowed the reverse pattern (striatal-cortical family, with 15 models in total, $\mathrm{M}_{4-18}$ ), and those that allowed modulations to both types of connections (both family, with 45 models in total, $M_{19-63}$ ). We found it very difficult to define the most likely locus of input a priori, given empirical evidence that both the striatum and the IPS receive inputs from sensory pathways (Saint-Cyr et al., 1990; Grefkes and Fink, 2005; Anderson et al., 2010; Reig and Silberberg, 2014; Vossel et al., 2014; Alloway et al., 2017; Guo et al., 2018). Instead, we opted to first determine whether the data were better modelled using the putamen or the IPS as the input. Importantly, this parameter did not vary over experimental conditions; therefore, this parameter did not explain changes in network activity that were attributable to the multitasking manipulation. We therefore implemented the full set of models $\left(M_{1-63}\right)$ with inputs to either the IPS, or to the putamen, so that we could test which input best explained the data (invariant to whether the input was from a single-task or multitask trial). Thus, we fit a total of $126(2 \times 63)$ models to the pre-practice data.

To make inferences regarding the modulatory influence of practice on both single and multitask conditions, we conducted the following for both the single-task and the multitask data (see Extraction of fMRI signals for DCM analysis section for details on data extraction): based on the endogenous connectivity and locus of driving input identified by the preceding analysis, we then fit the 
possible modulatory influences of the practice factor (i.e., pre-practice to post-practice).

\section{Extraction of fMRI signals for DCM analysis}

The brain ROls were selected by the following steps. First, we identified regions that showed increased activity for both single tasks at the pretraining session. Second, we sought which of these showed increased activity for multitask trials relative to single-task trials. Lastly, we asked which of these regions also showed a practice (pre vs post) by group interaction (Garner and Dux, 2015). The left and right IPS, left and right putamen, and the SMA were implicated by this interaction. In the interest of reducing the complexity of the model space, and in the absence of lateralized differences in the current data, we included only regions in the left hemisphere (LH) and the SMA in the first analysis.

For each region, we restricted the initial search radius by anatomically defined ROI masks and extracted the first eigenvariate of all voxels within a sphere of $4-\mathrm{mm}$ radius centered over the participant-specific peak for the initial contrast (increased activity for both single tasks, as in the previous study), adjusted for the effects of interest $(p<$ 0.05 , uncorrected). We opted to use this approach, rather than selecting a fixed functional $\mathrm{ROI}$ across participants, as we know that there are clear individual differences in the exact peak of BOLD signal changes within the brain regions that constitute the multiple demand network when participants perform comparable sensorimotor tasks (Crittenden and Duncan, 2014). Therefore, we sought to ensure that we identify the voxels for each participant that are most responsive to the functional localizer of interest, while leveraging a priori knowledge gained by the group-level contrasts $(N=100)$ and prior anatomic knowledge. This, in our opinion, utilizes a good combination of our a priori knowledge concerning brain structure and function, to localize meaningful BOLD signal changes at the individual level.

We created the anatomic masks in standard MNI space using FSL. As can be seen from Extended Data Figure 24 , participant-level peaks tended to cluster within the anatomically defined region, as would have occurred had we used a spherical ROI based on the functional data. For the IPS, we used the Juelich Histologic atlas, and for the putamen and the SMA, we used the Harvard-Oxford cortical and subcortical atlas. Note: to analyze the modulatory influence of practice on single-task data, we regressed out activity attributable to the multitask condition at this step. To analyze the modulatory influence of practice on multitask data, we comparably regressed out the singletask data at this step. For the first analysis concerning the multitasking network, we concatenated the six functional pretraining runs to form a single time series, and for the analysis of the influence of practice, we concatenated the six pretraining and six post-training runs (total runs $=12$ ). The two resulting time series were each adjusted for confounds using regressors for movement and for each run. The DCMs were fit using the resulting time series and hence provide a global estimate for the interactions among areas across the whole experiment rather than trial-specific estimates.
It is reasonable to expect that over the course of the experiment there will be some degree of variability between the flow of information from putamen to preSMA, because of potential fatigue and/or over learning effects. These nuisance effects are however mitigated in our event-related design.

\section{Bayesian model comparison and inference over parameters}

As our hypotheses concerned the modulatory influence of our experimental factors on model characteristics, rather than any specific model per se, we implemented random effects Bayesian model comparison between model families (Penny et al., 2010), with both family inference and Bayesian model averaging (BMA) as implemented in SPM 12. We opted to apply a random effects approach that uses a hierarchical Bayesian model to estimate the parameters of a Dirichlet distribution over all models, to protect against the distortive influence of outliers (Stephan et al., 2009). Specifically, the Dirichlet density describes the probability of each model, given the probability of all the models across the group. Its parameters can be considered as a proxy for a count for how many times a model won across participants. Therefore, improbable individual contributions to the group-level data are down-weighted proportional to the likelihood of the observation and contribute less to the evidence over the model space (e.g., a model that only wins for one participant will not hold much weight in the model probability space). This therefore mitigates the potential influence of individual outliers on the model selection procedures. For each family comparison, we report (1) the expectation of the posterior probability [i.e., the expected likelihood of obtaining the model family $k$, given the data $\left.p\left(f_{k} \mid Y\right)\right]$, and (2) the exceedance probability of family $k$ being more likely than the alternative family $j$, given the data $p\left(f_{k}>f_{j} \mid Y\right)$ (see Penny et al., 2010). To ensure that a particular family of models is not advantaged because of merely containing more models than a comparison family, a uniform prior needs to be set at the family level. The prior over a given family is defined as $p\left(f_{k}\right)=1 / K$, where $K$ is the total number of families. As the prior at the family level is obtained by summing the priors across constituent models in the family set, the uniform family prior is implemented at the model $(m)$ level as $p(m)=1 / K N_{k} \forall m \in f_{k}$, where $N$ is the number of models in family $k$ (Penny et al., 2010).

Upon establishment of the winning family, we sought to identify, post hoc, which specific parameters were likely, given the data, and when relevant, where there was evidence for group differences. To achieve this, we calculated the posterior probability $(\mathrm{Pp})$ that the posterior density over the given parameter has deviated from zero (or in the case of group differences, whether the difference between posterior estimates has deviated from zero), using the SPM spm_Ncdf.m function. To correct for multiple comparisons, we reported Pps as having deviated from zero when the likelihood exceeded that set by the Sidak correction $(1-\alpha)^{1 / m}$, where $\mathrm{m}=$ the number of null hypotheses being tested. 


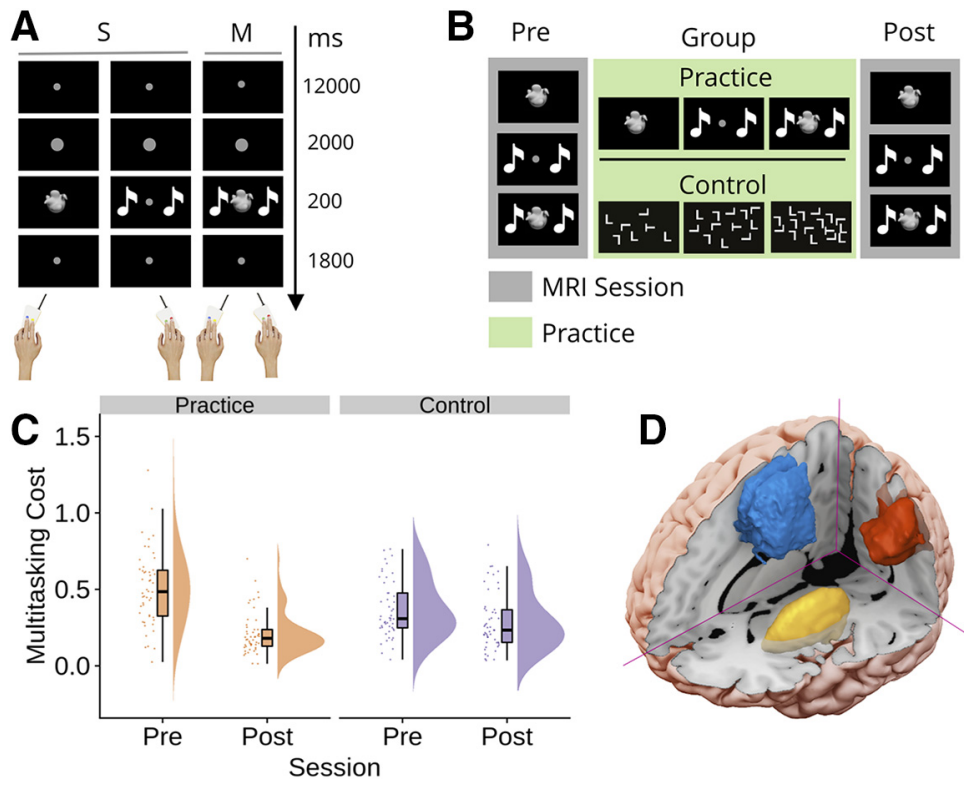

Figure 1. Task, protocol, behavior and ROls. A, Multitasking paradigm. The task comprised two single-task (S) conditions and one multitask (M) condition. Each S was a two alternative-discrimination between either one of two equiprobable stimuli. The stimuli could either be shapes (visual-manual task) or sounds (auditory-manual task). On M trials, participants were required to complete both Ss (visual-manual and auditory-manual). On all trials, participants were requested to perform the task(s) as quickly and as accurately as possible. B, Protocol. At both the pre-practice and post-practice sessions, all participants completed the multitasking paradigm while structural and functional MRI images were taken. Participants were then allocated to either the practice or the active-control group. The practice group subsequently performed the multitask paradigm over three sessions, whereas the control group practiced a visual-search task with three levels of difficulty, under a comparable reinforcement regimen. $\boldsymbol{C}$, Multitasking costs to RT [mean(Ms) - mean(Ss)] for the practice and control groups, at the pre-practice and post-practice sessions, presented as individual data points, boxplots, and densities (raincloud plots; Allen et al., 2018). D, ROls identified by our previous study (Garner and Dux, 2015); the SMA (blue), the IPS (red), and the putamen (yellow). The data from the $S$ and M conditions at the pretraining session are shown in Extended Data Figure 1-1.

\section{Results}

As all results unrelated to the DCM analysis are described in detail in Garner and Dux (2015), we recap the relevant findings here. Participants completed a multitasking paradigm (Fig. 1A) while being scanned with fMRI, in a slow event-related design. For the multitasking paradigm, participants completed both single-task and multitask trials. For the single-task trials, participants made a 2-AD between either one of two equiprobable shapes (visual-manual task) or between one of two equiprobable sounds (auditory-manual task). Participants were instructed to make the correct button-press as quickly and as accurately as possible. On multitask trials, the shape and sound stimuli were presented simultaneously, and participants were required to make both discriminations (visual-manual task and auditory-manual task) as quickly and as accurately as possible. Between the pre-practice and post-practice scanning sessions, participants were randomly allocated to a practice group or an active-control group (also referred to as the control group). The practice group performed the multitask paradigm over multiple days whereas the control group practiced a visual-search task (Fig. 1B). For both groups, participants were adaptively rewarded for maintaining accuracy while reducing RT (for details, see Materials and Methods).

Our key behavioral measure of multitasking costs was the difference in RT between the single-task and multitask conditions. Performing the component tasks as a multitask increases RT for both tasks, relative to when each is performed alone as a single task. The effectiveness of the paradigm to assess multitasking was confirmed with multitasking costs being clearly observed in the pre-practice session (main effect of condition, single-task vs multitask, $F_{(1,98)}=688.74$, mean square error $(\mathrm{MSE})=0.026, p<$ $0.0001, \eta_{\mathrm{p}}^{2}=0.88$; see Extended Data Fig. 1-1). Critically, the practice group showed a larger reduction in multitasking costs between the pre-practice and post-practice sessions than the control group [significant session (pre vs post) $\times$ condition (single-task vs multitask) $\times$ group (practice vs control) interaction; $F_{(1,98)}=31.12$, MSE $=0.01, p<0.001$, $\eta_{\mathrm{p}}^{2}=0.24$; Fig. 1C]. Specifically, the practice group showed a mean reduction (pre-cost - post-cost) of $293 \mathrm{~ms}(95 \% \mathrm{Cl}$ $[228,358])$, whereas the control group showed a mean reduction of $79 \mathrm{~ms}(95 \% \mathrm{Cl}[47,112])$. These findings did not appear to be due to a speed/accuracy trade-off as the group $\times$ session $\times$ condition interaction performed on the accuracy data were not statistically significant $(p=0.06)$.

We sought to identify the brain regions that could be part of the multiple demand network that supports performance of both tasks, as our question pertains to how regions that appear to be associated with cognitive control, invariant to the modality of the underlying tasks, interact under conditions of multitasking and multitasking practice. Specifically, ROls were defined as those that (1) 
showed increased activity for both single tasks (i.e., a conjunctive contrast), as could be expected by brain areas containing neurons that adapt to represent the current cognitive episode and brain areas that contribute to, or at least are sensitive to, the performance of both tasks; (2) showed sensitivity to multitasking demands (i.e., increased activity for multitask relative to single-task trials); and (3) showed specificity in response to the training regimen, i.e., showed a group $\times$ session interaction (for details, see Garner and Dux, 2015). Thus, our ROls are sensitive to both single tasks and to the multitasking practice regimen, regardless of laterality and the modality of the underlying single tasks. This criterion isolated the preSMA/SMA, the left and right inferior parietal sulcus (IPS) and the left and right putamen.

For the first analysis of the current study, in the interest of parsimony regarding the number of areas (nodes) in our models, and given that the current data suggested no strong reason to assume lateralized differences in the function of the currently defined underlying network, we opted to include only the pre-SMASSMA and the remaining LH regions as our ROls (Fig. 1D; Erickson et al., 2005, 2007; Dux et al., 2006, 2009; Filmer et al., 2013). Upon completion of this analysis, we then sought to understand which of our conclusions might be hemisphere specific. It is worth noting that we cannot conclusively infer whether any lateralized differences are because of genuine functional hemispheric differences or extraneous factors. However, such an analysis does provide insights into which conclusions can be drawn generally, regardless of hemisphere (and consequent decisions over the model space), and those which are hemisphere specific. To this end we repeated the analysis using the right hemisphere $(\mathrm{RH})$ regions as our ROls. We discuss the results of the $\mathrm{LH}$ analysis first, while referencing which findings did and did not generalize to the $\mathrm{RH}$. We then present the details of the findings from the $\mathrm{RH}$ analysis.

\section{Network dynamics underlying multitasking}

We sought to identify how multitasking modulates connectivity between the IPS, pre-SMA/SMA, and the putamen. Although our anatomically defined mask included all of SMA, the majority (78\%) of participants showed peak activity in pre-SMA, defined as coordinates rostral to the vertical commissure anterior in a probabilistic atlas based on resting state data from 12 participants (Kim et al., 2010). Moreover, a visual examination of the locations of the individual peaks suggest that the remaining $22 \%$ showed peak activity changes close to this probabilistic boundary (Extended Data Fig. 2-4). Therefore, we hereon refer to the region as pre-SMA (note: the within group percentages were also comparable; practice $=83 \%$, control $=73 \%$ ). To achieve this, we first applied DCM to construct hypothetical networks that could underlie the observed data. These models were then grouped into families on the basis of characteristics that addressed our key questions. This allowed us to conduct random effects family-level inference (Penny et al., 2010) to determine which model characteristics were most likely, given the data. Specifically, we asked: (1) which region drives inputs to the proposed network, invariant to the experimental multitasking manipulation (putamen or IPS family; Fig. 2A)? and (2) does multitasking modulate striatal-cortical couplings, corticocortical couplings or both (Fig. 2B)? Lastly, we conducted BMA within the winning family to make inference over which specific parameters were modulated by multitasking (i.e., is the posterior estimate for each connection reliably different from 0 ?)

The model space (Extended Data Fig. 2-1) which underpins our theoretically motivated hypothetical networks contained bidirectional endogenous connections between all three regions. Although effective connectivity can be investigated independently of anatomic connectivity, we selected this endogenous connectivity pattern given the extensive evidence for the existence of anatomic connections between the putamen, IPS, and pre-SMA (Alexander et al., 1986; Cavada and Goldman-Rakic, 1989; Luppino et al., 1993; Wise et al., 1997; Haber, 2016), as well as endogenous self-connections. As we had no a priori reason to exclude a modulatory influence of multitasking on any specific direction of coupling, we considered all 63 possible combinations of modulation (Extended Data Fig. 2-2).

First, we asked which region in the network received driving inputs that are modulated by multitasking demands. As the IPS shows sensitivity to sensory inputs across modalities (Grefkes and Fink, 2005; Anderson et al., 2010; Vossel et al., 2014), and as the striatum receives sensory-inputs from both the thalamus (Alloway et al., 2017) and from sensory cortices (Saint-Cyr et al., 1990; Reig and Silberberg, 2014; Guo et al., 2018), both IPS and putamen were considered as possible candidates. Given the distribution of probability over models, it is plausible (for example) that input arrives at both the IPS and the putamen. We decided to deal with this possibility by performing BMA over the most likely models. This allows us to capture information from models that allow inputs to either the putamen or the IPS, but only under circumstances where the evidence shows that neither input should be favored over the other. We opted to aggregate information this way as it allowed us to examine the impact of both inputs on the model evidence (separately) and retain parsimony over the model space. We therefore fit each of the 63 modulatory models twice, once allowing driving inputs to occur via the IPS, and once allowing input via the putamen [therefore, total models $\left.\left(\mathrm{M}_{\mathrm{i}}\right)=126\right]$. These models were grouped into two families, on the basis of their input [IPS input family ( $f_{\text {IPS }}$ ) and putamen input family $\left(f_{\text {Put }}\right)$. The evidence favored the putamen family [expected probability $\left(\mathrm{p}\left(\mathrm{f}_{\mathrm{Put}} \mid \mathrm{Y}\right)\right)$ : 0.54 , exceedance probability $\left(\mathrm{p}\left(\mathrm{f}_{\mathrm{Put}} \mid\right.\right.$ $Y>f_{\text {IPS }} \mid Y$ ): 0.79; Fig. $\left.2 A\right]$ relative to the IPS family. Therefore, the data are best explained by models where multitasking modulates driving inputs to the putamen. The winning putamen input family were retained for the next stage of family level comparisons. Note, for the $\mathrm{RH}$, the evidence did not disambiguate between input families. Therefore, we conclude that both subcortical and cortical inputs are likely to drive the network that underpins multitasking.

We then asked whether the data were better explained by models that allowed multitasking to modulate striatal- 

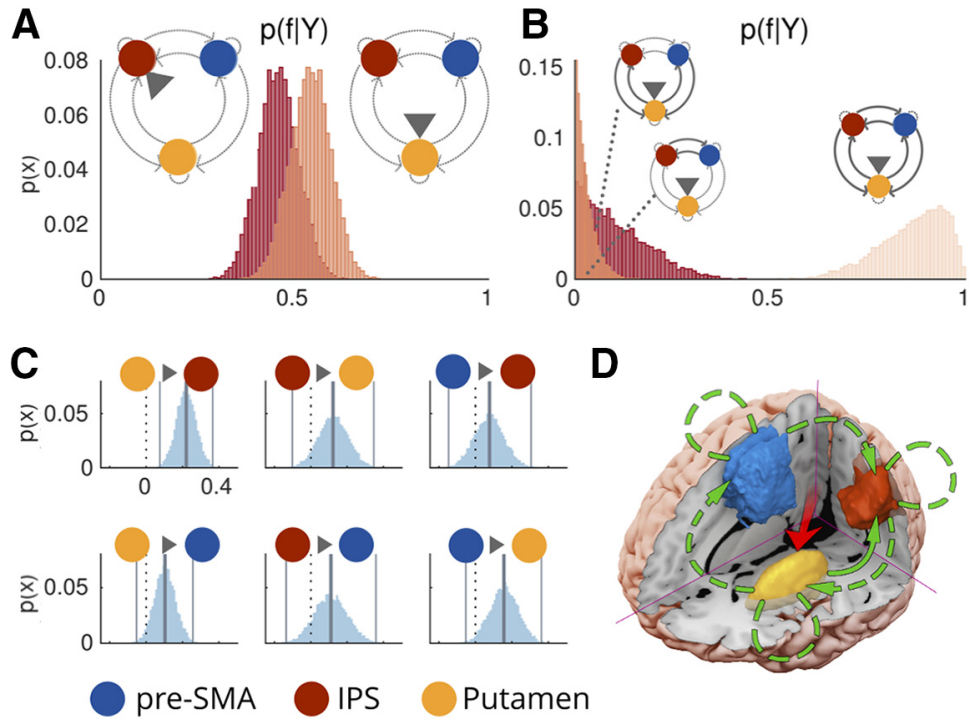

Figure 2. The modulatory influence of multitasking on the LH pre-SMA/IPS/putamen network. For the defined endogenous connections see Extended Data Figure 2-1. A, Posterior probabilities over families, given the data [p(f|Y)], defined by inputs to IPS (left distribution) or putamen (right distribution). The evidence favors driving inputs via putamen. B, Posterior probabilities over families differing in the connections modulated by multitasking (from left to right: corticostriatal modulations, corticocortical modulations, or both). Model evidence favors both corticostriatal and corticocortical couplings [for the posteriors over the endogenous (A) parameters; Extended Data Fig. 2-2). C, Posterior distributions over B parameters. Vertical lines reflect posterior means and 99th percentiles, whereas the dotted black line $=0$. Multitasking reliably increased modulatory coupling from the putamen to the IPS. $\boldsymbol{D}$, Proposed model for the modulatory influence of multitasking. Connections drawn with a continuous line denote significantly modu-

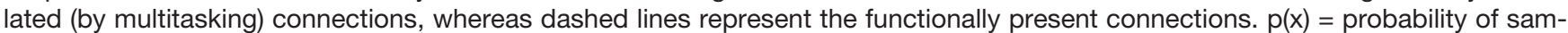
ple from posterior density. For the location of the individual peaks within each region, see Extended Data Figure 2-4.

cortical connections, corticocortical connections, or all (Fig. 2B). We therefore grouped the models from the putamen input family into three groups. The striatal-cortical family $\left(f_{\mathrm{SC}}\right.$ ) contained models that allowed multitasking to modulate any combination of the striatal-cortical connections, and none of the corticocortical connections. The corticocortical family $\left(f_{\mathrm{CC}}\right)$ contained models with the opposite pattern; multitasking could modulate any pattern of corticocortical couplings and none of the striatal-cortical couplings). Finally, in the all family, we considered models that included modulations to both striatal-cortical and corticocortical couplings $\left(f_{A L L}\right)$. In support of the idea that multitasking modulates striatal-cortical connectivity as well as corticocortical connections, the evidence favored the all family $\left[p\left(f_{A L L} \mid Y\right): 0.86, p\left(f_{A L L}\left|Y>f_{S C}, c C\right| Y\right)=1\right]$ over the striatal-cortical family $\left[p\left(f_{s c} \mid Y\right): 0.11\right]$ and the corticocortical family $\left[\mathrm{p}\left(\mathrm{f}_{\mathrm{cC}} \mid \mathrm{Y}\right)\right.$ : 0.03$]$. This result was also observed for the $\mathrm{RH}$.

Having determined that multitasking is indeed supported by both striatal-cortical and corticocortical couplings, we next sought to infer which specific parameters were modulated by multitasking; i.e., do we have evidence for bidirectional endogenous couplings between all regions? Or a subset of endogenous couplings? With regard to multitasking related modulations; are all couplings modulated, or a subset of striatal-cortical and corticocortical connections? To answer this, we conducted BMA over the all family to obtain the posteriors over each of the endogenous $(A)$ and modulatory coupling $(B)$ parameters. We looked for A parameters to retain by testing for which posteriors showed a probability of difference $(\mathrm{Pp})$ from zero that was $>0.992$ (applying the Sidak adjustment for multiple comparisons). As seen in Extended Data Figure 2-3, we retain endogenous couplings from IPS to Put, Put to IPS, Put to pre-SMA, and pre-SMA to IPS (all Pps =1) and reject endogenous couplings from IPS to pre-SMA $(\mathrm{Pp}=0.98)$ and pre-SMA to Put $(\mathrm{Pp}=0.66)$. In contrast, for the $\mathrm{RH}$, we retained all the endogenous connections.

We applied the same test to the B parameters and found evidence for a modulatory influence of multitasking on Put to IPS coupling $(\mathrm{Pp}=1)$. Although this specific result was not found for the $\mathrm{RH}$, we do find that IPS consistently shows multitasking induced coupling changes with other nodes (see the section of the RH analysis for details). Therefore, we conclude that the IPS appears to be a key node in modulating information flow through the network underpinning multitasking limitations, regardless of lateralization.

As opposed to the $\mathrm{RH}$, for the $\mathrm{LH}$ multitasking-induced modulation of putamen to pre-SMA coupling did not pass our rather strict threshold of $p=0.98$. However, it came very close. Specifically, the posterior distribution for this parameter did show reasonably strong evidence of multitask-induced modulations $(P p=0.96)$, the variance of this distribution was more similar to the retained than the rejected coupling parameters ( $\sigma=0.06$ vs $\sigma=0.08$ ), and unlike the rejected parameters, this connection showed strong evidence for the endogenous coupling $(P p=1$; Fig. $2 C)$. Furthermore, looking ahead to the $\mathrm{RH}$ analysis, we find further evidence that this coupling is modulated by multitasking demands. We therefore conclude that there is reasonable evidence that putamen to pre-SMA 


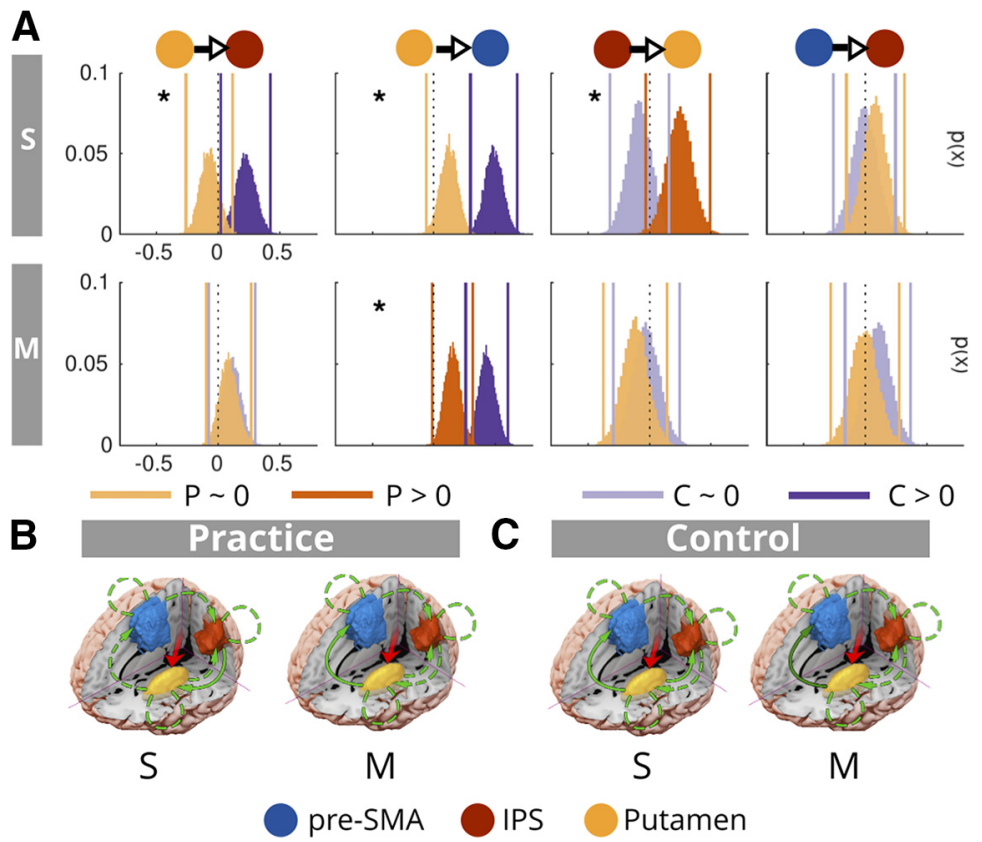

Figure 3. The modulatory influence of practice on the multitasking network. $\boldsymbol{A}$, Posteriors over parameters were estimated for the practice ( $\mathrm{P}$; in orange) and control (C; in violet) groups for single-task trials $(\mathrm{S})$ and for multitask trials (M). Posteriors that deviated reliably from $0(>0)$ are in darker shades, whereas those that did not significantly deviate from 0 are in lighter shades. Asterisks indicate where there were statistically significant group differences. Proposed influences of practice on modulatory coupling within the multitasking network for single-task (S) $\boldsymbol{B}$ and multitask (M) $\boldsymbol{C}$ trials, for the practice and control groups. For multitask trials, the arrows are shaded to indicate the strength of the effect (i.e., the darker the arrow, the larger the modulation to that parameter between groups). For the model space considered in this analysis, see Extended Data Figure 3-1. For the expected and exceedance model probabilities, see Extended Data Figure 3-2.

coupling is modulated by multitasking. We reject a modulatory influence of multitasking on the remaining parameters (all Pps $\leq 0.88$ ).

To sum up (Fig. 2D), the influence of multitasking is best explained in the LH by a network where information is propagated, via Putamen (Put), to the IPS and the preSMA. Information is shared back to the Put via IPS, and from pre-SMA to IPS. Overall, we conclude that multitasking demands specifically increases the rate of information transfer sent from Put to pre-SMA, and between the IPS and other subcortical and cortical nodes. Hence, we can reject the idea that multitasking costs are solely because of limitations in a cortical network, rather they also reflect the taxation of information sharing between the Put and the other relevant cortical areas, namely, the IPS and the pre-SMA.

\section{The influence of practice on the network underpinning multitasking}

Next, we sought to understand how practice influences the network that underpins multitasking on both singletask and multitask trials, for both the practice and control groups. For example, it may be that practice influences all the endogenous couplings in the network or a subset of them. Furthermore, if practice only modulated a subset of couplings, would it only be striatal-cortical couplings, or corticocortical, or both? By comparing the practice group to the control group, we sought to identify which modulations are because of engagement with a multitasking regimen, and which are because of repeating the task only at the post-session (and potentially because of engagement with a practice regimen that did not include multitasking). To address these questions, we constructed DCMs that allowed practice (i.e., a pre/post session factor) to modulate all the possible combinations of couplings in the multitasking network defined above (four possible connections, therefore $M_{i}=15$; Extended Data Fig. 3-1). We then fit these DCMs separately to the singletask data and to the multitask data, concatenated across pre to post sessions. Comparable to above, we decided to leverage information across models (proportional to the probability of the model; Extended Data Fig. 3-2) and conducted random-effects BMA across the model space to estimate posteriors over the parameters. This method can be more robust when the model space includes larger numbers of models that share characteristics, as it helps overcome dependence on the comparison set by identifying the likely features that are common across models (Penny et al., 2010). We compare the resulting posteriors over parameters to determine for each group, those which deviate reliably from zero for single-task trials, for multitask trials, and also whether they differ between groups (applying the Sidak correction for each set of comparisons).

The results from the analysis of posteriors over parameters can be seen in Figure 3. Findings that showed some generalization in the subsequent $\mathrm{RH}$ analysis are as follows; for single-task trials, in the practice group, the practice factor modulated coupling from IPS to Put ( $P p=0.99$, 
$>0.987$, Sidak adjustment for multiple comparisons), which was also larger than that observed for the control group (Pp practice $>$ control $=0.99$ ). This was partially observed in the $\mathrm{RH}$ (see the $\mathrm{RH}$ analysis below for details). For the practice group, no other modulatory couplings achieved the criteria for significance (all Pps $\leq 0.96$ ). For the control group, the practice factor modulated Put to pre-SMA couplings $(P p=1$, replicated in the $\mathrm{RH}$, but for multitask trials), and the influence of practice was larger on this coupling for the control group than for the practice group (Pps control $>$ practice $=0.99$ ). Practice also modulated Put to IPS coupling $(P p=0.99)$, and this modulation was larger for the control than the practice group $(P p=1)$. For multitask trials, both groups showed practice related increases to modulations of the putamen to preSMA coupling (practice group $P p=0.99$, control $P p=1$, also see the $\mathrm{RH}$ analysis, where this was observed for the control group on multitask trials). Perhaps counterintuitively, these were larger for the control group than for the practice group (Pp control > practice $=1$ ); note: we dissect this relationship further in the discussion. The remaining modulatory parameters and group differences did not achieve statistical significance (all Pps $\leq 0.93$ ). Overall, practice largely influences coupling changes between putamen and IPS, and putamen and pre-SMA, and this is observed for both hemispheres (some additional coupling changes observed specifically for the $\mathrm{RH}$, which are discussed in $\mathrm{RH}$ analysis).

\section{Interrogating the $\mathrm{RH}$ to determine lateralization of effects}

Focusing on the $\mathrm{LH}$ afforded a reduced model space while also eschewing overparameterized models. However, given that there are known hemispheric asymmetries in the brain, it is reasonable to wonder which of these results would hold, had we considered the $\mathrm{RH}$. To establish which of our conclusions would have still been reached had we not used LH ROls, we repeated the above analyses, this time using the RH data (right IPS, right Put, and pre-SMA). First, we report the details of the commonalities between the $\mathrm{LH}$ and $\mathrm{RH}$ analyses and then report the specifics of the differences. Comparable to the LH analysis, practice increased the strength of putamen to pre-SMA coupling on multitask trials, however this time we observed it only for the control group $(\mathrm{Pp}=1)$, and not for the practice group $\left(\mathrm{Pp}=0.79, \alpha_{\mathrm{SID}}=0.991\right)$, although we also did not detect a difference between the two groups $\left(\mathrm{Pp}=0.97, \alpha_{\mathrm{SID}}=0.991\right)$. Therefore, we suggest that multitasking influences information transfer from putamen to cortex, and that this occurs for both hemispheres.

Some lateralized differences must also be noted. For the multitasking network, in contrast to the LH model, evidence favored neither input family [expected probability $\left(p\left(f_{\text {Put }} \mid Y\right)\right): 0.5$, exceedance probability $\left(p\left(f_{\text {Put }}\left|Y>f_{I P S}\right| Y\right)\right.$ : $0.48]$; therefore, the models from both input families were included in the subsequent connection family comparison. Comparable to the LH model, the evidence favored the all family $\left.\left[p\left(f_{A L L} \mid Y\right): 0.89, p\left(f_{A L L}\right) Y>f_{S C}, c C \mid Y\right)=1\right]$ over the striatal-cortical family $\left[\mathrm{p}\left(\mathrm{f}_{\mathrm{Sc}} \mid \mathrm{Y}\right): 0.09\right]$ and the corticocortical family $\left[p\left(f_{c c} \mid Y\right): 0.02\right]$. Unlike the LH model, posterior estimates obtained over parameters using BMA provided evidence to retain all the endogenous connections (all Pps =1). Whereas multitasking did not modulate corticocortical connections in the $\mathrm{LH}$, there was evidence that multitasking modulates RH pre-SMA $->$ IPS, and IPS $->$ pre-SMA coupling (both $\mathrm{Pps}=1$, all remaining Pps $<0.98)$. This suggests that multitasking exerts greater influence on cortical couplings in the $\mathrm{RH}$ than the $\mathrm{LH}$, and that our conclusions are in part sensitive to the hemisphere from which we select our ROIs. Importantly, and as mentioned above, the observation that multitasking modulates putamen to pre-SMA coupling is consistent across hemispheres, thus demonstrating convergent evidence that multitasking limitations stem, at least in part from modulations in information transfer between these two nodes of the network.

As the winning $\mathrm{RH}$ network underpinning multitasking contained all 6 endogenous connections, we considered all 63 possible combinations of modulation for the practice factor (Extended Data Fig. 2-2). Additionally, and because of the absence of evidence favoring either input, we included models that allowed inputs to the IPS and those that allowed inputs via Put $\left(T_{m}=126\right)$. As this constitutes the full model space, our goal was to first determine whether we could exclude families of models, before performing BMA to obtain subject level posterior estimates over parameters. We therefore conducted the same family comparisons as reported above for the multitasking network analysis. For single-task trials, and in contrast to the LH, models allowing inputs to the IPS were favored over those with inputs via Put [expected probability $\left(p\left(f_{I P S} \mid Y\right)\right): 0.57$, exceedance probability $\left(p\left(f_{I P S} / Y>f_{\text {Put }} \mid Y\right)\right.$ : 0.89)], whereas for multitask trials, the evidence was far less conclusive [expected probability $\left(p\left(f_{\mathrm{IPS}} \mid \mathrm{Y}\right)\right)$ : 0.51 , exceedance probability $\left(p\left(f_{\mid P S}\left|Y>f_{\text {Put }}\right| Y\right)\right.$ : 0.58$\left.)\right]$. Therefore, for single trials, we retained only the $f_{I P S}$ for the next stage of the analysis, whereas all models were retained for multitask trials. As we sought to conduct BMA over the winning family for each group separately in the next stage of the analysis, we split the practice and control group data before making model family comparisons based on connectivity patterns. For both groups, and for both single-task and multitask trials, evidence favored the all family [all $p\left(f_{A L L}\left|Y>f_{S C}, c C\right| Y\right)>0.99$; Fig. 4].

Examination of the posterior estimates over parameters revealed some differences in the specific couplings modulated by practice for each group. In contrast to the LH analysis, we did not find that practice modulates putamen and IPS coupling, although we did find that practice modulated IPS -> pre-SMA coupling for both the control group on single-trials $(P p=1)$ and the practice group on multitask trials $(\mathrm{Pp}=1$; Fig. 5), suggesting that regardless of laterality, the IPS is a key site for practice-induced network changes. Importantly, for both the $\mathrm{LH}$ and $\mathrm{RH}$ analysis, we detect a practice related modulation on Put to pre-SMA coupling, showing that this conclusion is robust to both hemispheric and model specifications.

\section{Signal comparisons between $\mathrm{LH}$ and $\mathrm{RH}$, and interregional correlations}

It may at first appear counterintuitive that we find some differing results between the $\mathrm{LH}$ and $\mathrm{RH}$. We know from 

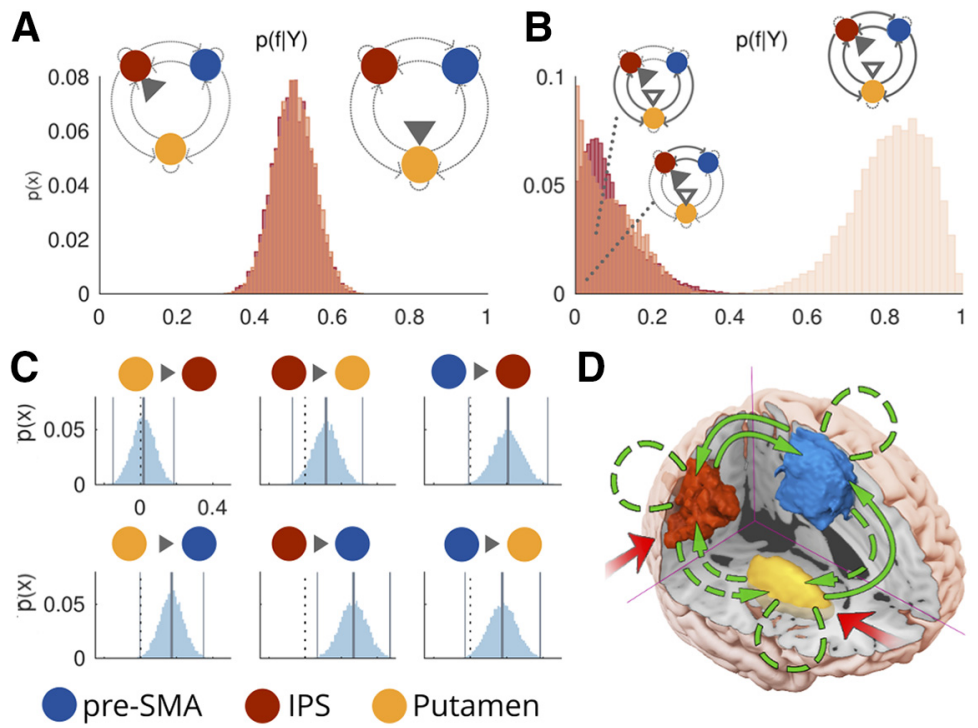

Figure 4. The modulatory influence of multitasking on the $\mathrm{RH}$ network (see control analysis section). $\boldsymbol{A}$, Posterior probabilities over families, given the data $[\mathrm{p}(\mathrm{f} \mid \mathrm{Y})]$, defined by inputs to IPS (left distribution) or putamen (right distribution). B, Posterior probabilities over families differing in the connections modulated by multitasking (from left to right: corticostriatal modulations, corticocortical modulations, or both), averaged across families with input to either IPS or putamen. $\boldsymbol{C}$, Posterior distributions over B parameters. Vertical lines reflect posterior means and 99th percentiles, whereas the dotted black line $=0$. $\boldsymbol{D}$, Proposed model for the modulatory influence of multitasking in the $\mathrm{RH} . \mathrm{p}(\mathrm{x})$ = probability of sample from posterior density.

our original analysis (Garner and Dux, 2015) that our ROls from both hemispheres interact with our experimental factors (i.e., show a group $\times$ session $\times$ condition interaction). We have confidence in these results owing to our large sample size $(N=100)$, and suitable corrections for multiple comparisons. As DCM models BOLD responses using a GLM, with the addition of a forward model that projects GLM parameters to a predicted BOLD response (Friston
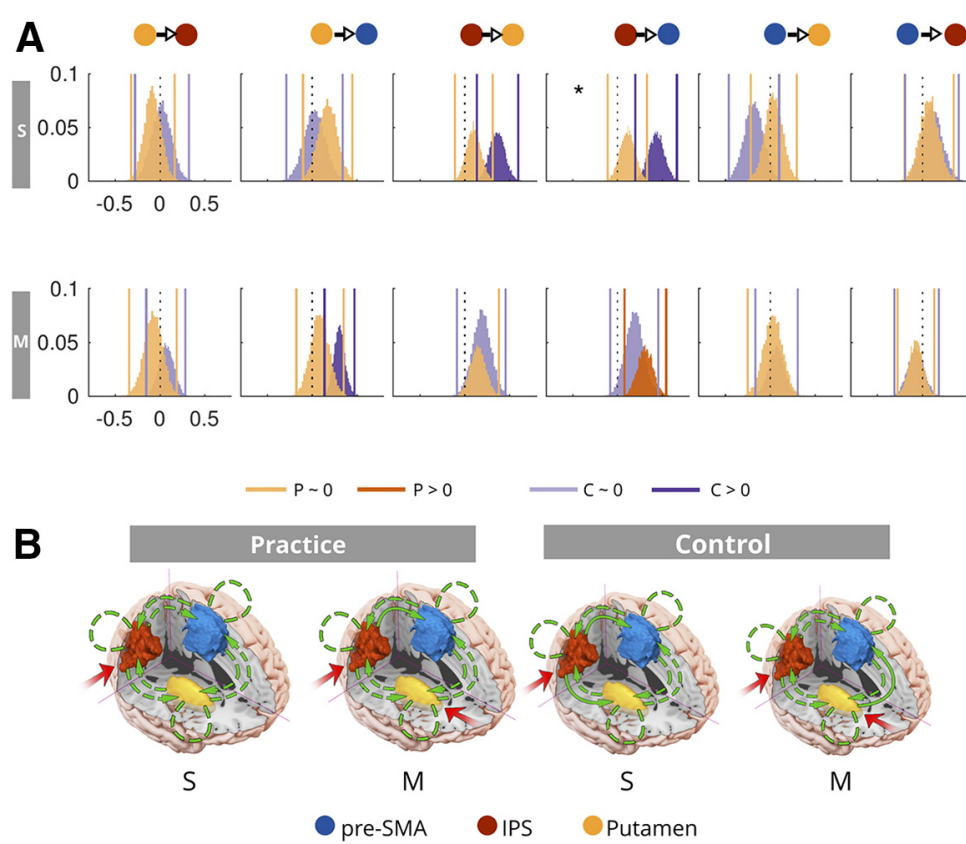

Figure 5. A, Showing group-level posteriors over parameters estimated for the practice (P, in orange) and control $(C$, in violet) groups for single-task trials $(\mathrm{S})$ and for multitask trials $(\mathrm{M})$. Posteriors that deviated reliably from $0(>0)$ are in darker shades, whereas those that did not significantly deviate from 0 are in lighter shades. Asterisks indicate where there were statistically significant group differences. $\boldsymbol{B}$, Proposed influences of practice on modulatory coupling within the multitasking network for single-task (S) and multitask (M) trials, for the practice and control groups. For the posterior probabilities over family comparisons conducted for this knowledge, see Extended Data Figure 5-1. 
A

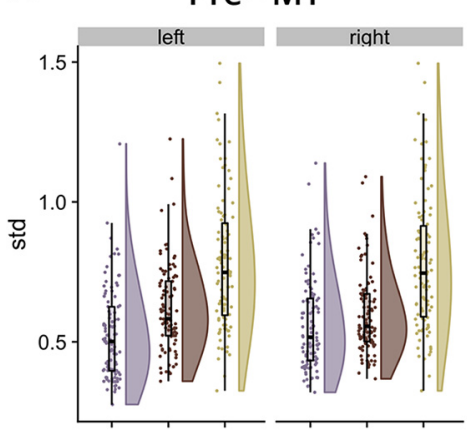

C

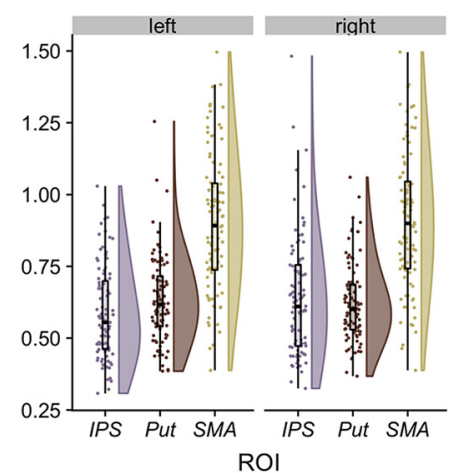

B

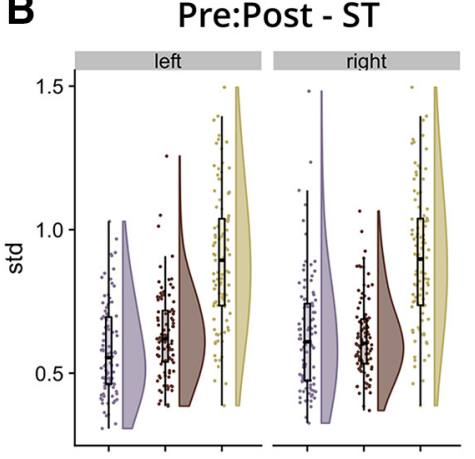

Figure 6. Showing the densities, boxplots and individual data points of the SDs of the mean centered time courses, across subjects, for the session 1 data (panel $\boldsymbol{A}$ ), the Pre-Post single-task data (panel $\boldsymbol{B}$ ) and the Pre-Post multitask data (panel $\boldsymbol{C}$ ). Data are shown for each ROI ( $x$-axis) for the LH (left) and the RH (right). ST = single-task, MT = multitask.

et al., 2003), we are well placed to use DCM to model our current dataset.

Albeit with a robust motivation, it is still useful to conduct some basic checks to inform whether the observed hemispheric differences are because of noise or are likely to be produced by genuine lateralized differences in function, particularly when they have the capability to shed insight into how robust the current observations may be. We therefore considered what analyses we could conduct to instill such confidence (or skepticism) in the current finding of hemispheric differences in the results of the DCM analysis. As DCM serves to model the time series data from each ROI, we reasoned that it is sensible to check the noisiness of the data underlying our current DCM analysis. We assume that a noisier time series would be more difficult to model, or indeed, may motivate overfitting of the models to the data (Lever et al., 2016). We therefore checked the "noisiness" or variance of our time course data across participants, to determine whether differences between the two hemispheres could be driven by noise differences, rather than signal differences. As each time course is mean centered and adjusted for effects of no interest, we opted to take the SD of the time course as a proxy of noisiness, rather than a signalto-noise ratio. We therefore computed the SD of each time course for each participant, region, hemisphere and DCM analysis (pre, single-task pre-post, multitask prepost). The results are presented in Figure 6. For each region, and time course and analysis, we compared the distributions between hemispheres (e.g., left IPS vs right IPS) using the $z$ score tests. All comparisons were not significant ( $z$ range: $[-0.3,0.12]$, all $p s>0.75)$. Therefore, the variance in the signal across participants was broadly comparable between $\mathrm{LH}$ and $\mathrm{RH}$, suggesting that the observed differences are less likely to be driven by random noise.

A second possible approach, suggested during the review process, would be to apply a simple correlation analysis between interregional time series data to see whether the observed statistical dependencies reflect what was observed for the DCM analysis, for example, do correlations between putamen and pre-SMA increase in strength as a function of multitasking demands or practice? The caveats for applying such an analysis are as follows: correlation and DCM analyses do not test the same relationships in the data. Correlative measures test for statistical dependencies in the signal, i.e., to what extent is time series $x$ associated with time series $y$ ? In contrast, DCM examines effective connections in the data, i.e., it asks at what rate does the theoretical neural source of time series $x$ have to affect the theoretical source of time series $y$, to generate the best match to the observed data? Indeed, this fundamental difference has been shown to yield dissociative effects. For example, autoregressive coefficients between two time series can be high, even in the absence of a direct effective connection (David et al., 2008; Friston et al., 2014). Thus, even if the correlation analysis were to show different relationships relative to the DCM analysis, we would be unable to draw definitive conclusions regarding the viability of the latter analysis. 
A

B

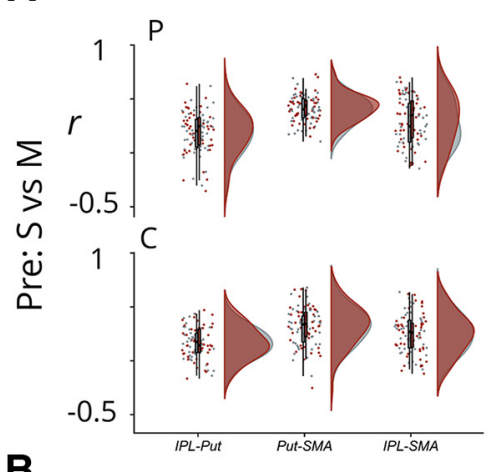

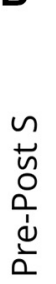

C

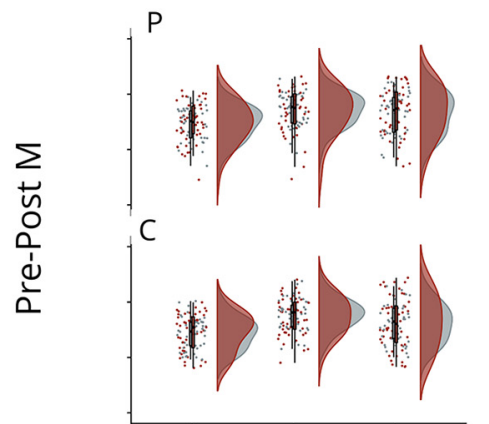

Right

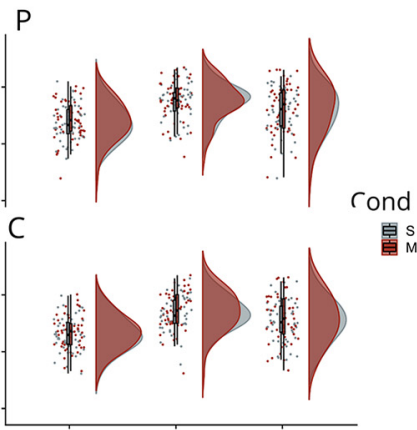

$\mathrm{P}$
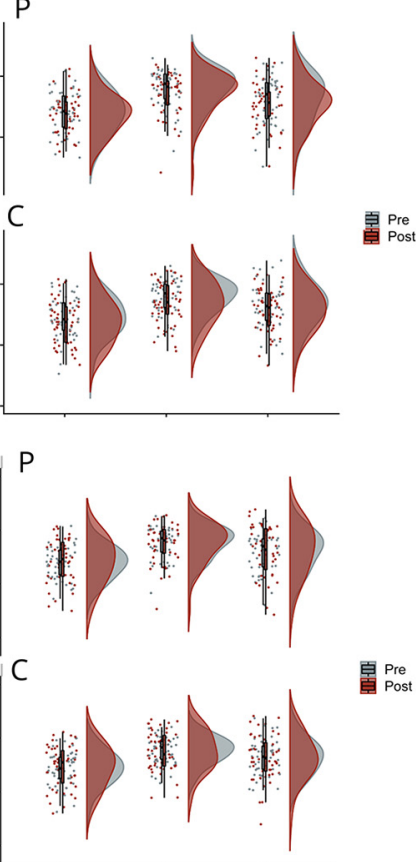

Figure 7. Showing the densities, boxplots and individual Pearson $r$ values obtained between each pair of regions ( $x$-axis), for each condition of interest. $\boldsymbol{A}$, Single (S) versus multitask (M) comparison. B, Pre-Post single-task data (S). C, Pre-Post multitask (M) data. Data shown for the $\mathrm{LH}$ (left) and the $\mathrm{RH}$ (right) separately. $\mathrm{P}=$ practice group, $\mathrm{C}=$ control group.

Although this does not provide a genuine sanity check for the DCM analysis, we present this analysis as a means of comparison for the interested reader.

For each hemisphere (left or right) and analysis (pre practice S v M, pre-post S, and pre-post M), we extracted the mean centered time course data for each participant and $\mathrm{ROI}$ and concatenated the time points relevant to each condition. For example, for the pre practice $S \vee M$ task, we concatenated all the time points that mapped to the $\mathrm{S}$ regressor in the GLM defined for the DCM analysis and repeated the process for time points that mapped to the $\mathrm{M}$ regressor. For each participant and condition, we correlated the time series between each possible pairing from our three ROIs (IPL-Put, Put-SMA, IPL-SMA), thus obtaining an $r$ value for each participant, condition, and region pair. The resulting $r$ values were then entered into the relevant second-level statistical comparison. For each analysis, we applied the Sidak adjustment for multiple comparisons.

\section{Pre-practice S v M data}

For the LH, although correlations for each interregion pairing numerically increased in the multitask condition, relative to the single-task condition (Fig. 7), none of these comparisons were statistically significant (all $t \mathrm{~s}_{(95)}<1.98$, all $p s \geq 0.05$ ). In contrast, for the $\mathrm{RH}$, we observed a statistically significant increase for multitask, relative to single-task correlations for the IPL-SMA pairing $\left(t_{(97)}=\right.$ -2.71, $p=0.008$ ), whereas the other two comparisons were not statistically significant $\left(t s_{(97)} \leq 1.92, p s \geq 0.058\right)$. This is in accordance with the observation yielded from the DCM analysis that multitasking modulates coupling between these two regions.

\section{Pre-post S}

For each hemisphere and region pair, a two (group: practice vs control) $\times$ two (condition: pre vs post) mixed ANOVA was applied to compare the time series correlations for 
single-task trials. For both hemispheres, there was a statistically significant decrease in the correlation size observed between pre-practice and post-practice for the Put-SMA pairing, $\left(\mathrm{LH}: F_{(1,93)}=11.96, \mathrm{MSE}=0.13\right.$, $p=0.0008$, pre $S r$ mean: 0.39, SE: 0.02, post $S r$ mean: 0.34, SE: 0.02, $\mathrm{RH}: F_{(1,92)}=10.44, \mathrm{MSE}=0.12, p=0.002$, pre $S r$ mean: 0.4 , SE: 0.01 , post $S r$ mean: 0.35 , SE: 0.02 ), which converges with our conclusion that practice modulates coupling between these brain regions. For the $\mathrm{RH}$, and again, in concert with the observations yielded by the DCM analysis, we observe a significant decrease in correlation strength for the IPL-SMA pairing $\left(F_{(1,92)}=7.34, p=0.008\right.$, pre $S r$ mean: 0.32 , SE: 0.02 , post $\mathrm{S} r$ mean: $0.28, \mathrm{SE}=0.02)$. No other comparisons were statistically significant (all $p s>0.1$ ).

\section{Pre-post M}

For both hemispheres, no comparisons were statistically significant (all $F_{\mathrm{s}_{(1,90)}}<1.3$, all $\left.p \mathrm{~s}>0.2\right)$.

\section{Discussion}

We sought to understand how multitasking demands modulate underlying network dynamics and how practice changes this network to reduce multitasking costs. We adjudicated between previously hypothesized models posing that multitasking demands modulate connectivity within (1) a frontal-parietal cortical network (Jiang, 2004; Marois and Ivanoff, 2005; Dux et al., 2006; Erickson et al., 2007; Sigman and Dehaene, 2008; Hesselmann et al., 2011; Tombu et al., 2011; Watanabe and Funahashi, 2014; Marti et al., 2015); or (2) or a striatal-cortical network (Badre and Nee, 2018; Caballero et al., 2018; Yartsev et al., 2018). We demonstrate evidence in keeping with the latter. Specifically, having previously identified that practice-related improvements correlate with activity changes in the pre-SMA, the IPS, and the putamen (Garner and Dux, 2015), we applied DCM to ask how multitasking and practice modulates connectivity between these regions. Regardless of whether we analyze ROIs from the $\mathrm{LH}$ or $\mathrm{RH}$, we observe that multitasking consistently modulates striatal-cortical connectivity, and most consistently, information transfer from putamen to preSMA. Therefore, multitasking appears to modulate information sharing within a broader network than has been implied by previous studies focusing on cortical brain regions only. Rather, our results accord with models of single-task decision-making implicating a distributed striatal-cortical network. Our results build on this work by specifically showing that attempting to multitask bilaterally increases rates of information sharing from putamen to the IPS and pre-SMA (among other modulations), and we propose that practice overcomes multitasking costs by alleviating taxation on information transfer from putamen to pre-SMA.

\section{Network dynamics underpinning cognitive performance in multitasking, implications}

We found that during multitasking, the currently interrogated network is driven by inputs to the putamen ( $\mathrm{LH}$ and
$\mathrm{RH}$ ), and likely also the IPS (RH). While information is propagated between cortical and subcortical areas, multitasking most consistently modulates coupling from the putamen to pre-SMA. Moreover, although multitasking and practice consistently modulates the coupling between the IPS and the other nodes of the network, exactly which node appears to be dependent on the hemisphere under interrogation (putamen for LH and pre-SMA for the $\mathrm{RH})$.

The IPS is assumed to contribute to the representation of stimulus-response mappings (Bunge et al., 2002; Goard et al., 2016; Pho et al., 2018), and the pre-SMA is assumed to arbitrate between competitive representations of action plans (Nachev et al., 2007). Thus, both regions potentially constitute key nodes in the cortical representation of current and upcoming stimulus-response conjunctions. Given that we observed consistent evidence that putamen to pre-SMA coupling is modulated by multitasking and practice, we propose that multitasking limitations stem, at least in part, from constraints on the rate at which the striatum can, on the basis of incoming sensory information, sufficiently excite the appropriate cortical representations of stimulus-response mappings to reach a threshold for action. This leads to the intriguing possibility that previous observations that cognitive control operations are underpinned by a frontal-parietal network (Dux et al., 2009; Cole et al., 2013; Duncan, 2013; Watanabe and Funahashi, 2014) may actually have been observing the cortical response to striatally mediated excitatory signals. In fact, our findings are in line with a recent application of meta-analytic connectivity modeling showing that when frontal-parietal regions associated with cognitive control operations are used as seed regions, the left and right putamen are likely to show significant co-activations across a range of sensorimotor and perceptual tasks (Camilleri et al., 2018). Taken together, these data suggest that the striatum, or at least the putamen, should be included in the set of brain regions that contribute to cognitive control, at least during sensorimotor decision-making and multitasking.

It was perhaps surprising that the best network only received inputs via the putamen when the analysis was conducted on the LH data, and via both the putamen and the IPS with the $\mathrm{RH}$ data. However, given that a right-lateralized network incorporating the IPS has been implicated in the reorienting of attention to less probable stimuli (Vossel et al., 2012), it may be that the second stimulus on multitask trials, which only occurred on one third of the trials, engaged the network that responds when events demand reorientation to a new sensory input. Furthermore, although the RH IPS showed multitasking-induced and practice-induced modulations of coupling with the preSMA, the lack of consistency between groups and hemispheres suggest that coupling activity between these nodes does not necessarily reflect the bottleneck of information processing that gives rise to multitasking costs.

\section{Implications of practice induced plasticity in remediating multitasking costs}

Here, we can both model the dynamics of the network that underpins multitasking limitations, and also identify 
which connections change with practice, for both singletasks and for multitasks. By comparing this to modulations observed in the control group, we can make inroads to identifying which couplings not only correspond to multitasking limitations, but also those that may be critical in determining the extent of their presence and remediation because of practice. We interpret the control group as showing modulations that occur as a consequence of being at an earlier stage of practice (i.e., repeating the task for a second time after having practiced a regimen not expected to improve multitasking; Garner et al., 2014, 2016; Verghese et al., 2018). In contrast, the practice group is at a longer-term stage of practice (i.e., they are repeating the task for the fifth time). It may appear counterintuitive that we observed modulations for the active control group from pre to post. However, merely repeating a task without any intervening practice is sufficient to produce performance gains (Boot et al., 2013). Moreover, we have observed these behavioral effects in our other practice studies (Garner et al., 2014, 2015; Verghese et al., 2018) and in the current study. Importantly, here and elsewhere, practice groups show larger behavioral benefits relative to such control groups.

In light of this framework, putamen to pre-SMA coupling appears to be consistently modulated by both shortterm and long-term practice (the latter being more evident in the LH). We found that being at an earlier stage of practice corresponds to an increase in the rate of information transfer from putamen to pre-SMA and that later stages are also associated with an increase of information transfer between these regions (in the LH) but to a lesser extent than is observed for short-term practice. We interpret these results as reflecting a trajectory of practice induced changes in putamen to pre-SMA coupling. Namely, repeating a task increases putamen to pre-SMA information transfer. We speculate that extended practice results in decreased requirement for faster information transfer between these two regions, potentially reflecting decreased reliance on this pathway with extended practice. Rodent studies consistently demonstrate that when a task is novel, firing in the dorsolateral striatum corresponds to the full duration of a trial. As the behavior becomes habitual, firing patterns transition to coincide with the beginning and end of chunked action sequences (Jog et al., 1999; Barnes et al., 2005; Jin and Costa, 2010; Thorn et al., 2010; Smith and Graybiel, 2013). These results imply a novel physiological substrate for the amelioration of multitasking limitations; namely, the duration of information transfer from putamen to pre-SMA during task performance.

\section{Further considerations}

It is worthwhile considering why the previous fMRI investigations into the neural sources of multitasking limitations did not implicate a role for the striatum. As far as we can observe, our sample size, and thus statistical power to observe smaller effects, is substantially larger than previous efforts (Szameitat et al., 2002, 2006; Jiang, 2004; Jiang et al., 2004; Dux et al., 2006, 2009; Marois et al., 2006; our $N=100$, previous work $N$ range: 9-35, Stelzel et al., 2006; Erickson et al., 2007; Sigman and Dehaene, 2008; Borst et al., 2010; Hesselmann et al., 2011; Tombu et al., 2011; Nijboer et al., 2014). One fMRI multitasking study has reported increased striatal activity when there is a higher probability of short temporal overlap between tasks (Yildiz and Beste, 2015). Moreover, meta-analytic efforts into the connectivity of the frontal-parietal network during cognitive control tasks implicate the putamen (Camilleri et al., 2018). Lesions of the striatum and not the cerebellum have been shown to correspond to impaired multitasking behaviors (Thoma et al., 2008), and intracranial EEG has revealed that fluctuations in oscillatory ventral striatal activity predicts performance on the attentional blink task (Slagter et al., 2017); a paradigm which is assumed to share overlapping limitations with those revealed by sensorimotor multitasks (Jolicoeur, 1998; Arnell and Duncan, 2002; Zylberberg et al., 2010; Tombu et al., 2011; Garner et al., 2014; Marti et al., 2015). Therefore, our findings do converge with more recent efforts that do indeed implicate a role for the striatum in cognitive control. We extend these findings to demonstrate how the striatum and cortex interact to both produce and overcome multitasking limitations.

Of course, we have only examined network dynamics in a few areas of a wider system that correlates with multitasking (Garner and Dux, 2015), and we are unable to know whether we observe an interaction in these specific regions because the interaction exists nowhere else, or because the interactions are more readily observable between these regions. Indeed, we have also observed in the current dataset that the volume of the rostral dorsal lateral prefrontal cortex inversely correlates with multitasking improvements in the practice group (Verghese et al., 2016). However, in this and our previous work (Garner and Dux, 2015), functional activation of the DLPFC did not meet criteria for inclusion in our analysis of the functional data. In the current study, we utilized simple sensorimotor tasks. The networks underpinning the translation of more complex sensorimotor mappings may well invoke more reliable functional activity in anterior ROls than we observed here (Dux et al., 2006; Woolgar et al., 2011; Crittenden and Duncan, 2014; Badre and Nee, 2018). Therefore, future work should determine whether more complex stimulus-response mappings would yield evidence warranting the addition of more anterior regions, such as the DLPFC, to the currently defined network.

With the current analysis, we sought to maintain parsimony by reducing the model space (and parameters) to one hemisphere, and to ascertain the findings we would have drawn regardless of which hemisphere was under interrogation. Given the bilateral nature of the observations reported in our previous work, yet the precedents in the literature for a $\mathrm{LH}$ bias in the networks underpinning multitasking (Erickson et al., 2005, 2007; Dux et al., 2006, 2009; Filmer et al., 2013), we were agnostic as to whether or not we should hypothesize differences between the two hemispheres. Our analysis approach enabled us to determine that multitasking limitations and their practice-related remediation are consistently related to changes in putamen to pre-SMA coupling, regardless of which hemisphere (i.e., which subset of the reduced model 
space) is selected for study. It remains a little more challenging to interpret the findings that were specific for each hemisphere. Given the questions of lateralization of function raised by the current and previous work, a principled and systematic investigation is warranted. For example, future work could simulate fMRI data in the absence and presence of genuine lateralized differences, to observe the sensitivity and robustness of DCM under these differing conditions. This simulation approach could also be applied to other degrees of freedom in the DCM analysis process. For example, we opted to use for each participant the $\mathrm{ROI}$ within the anatomic $\mathrm{ROI}$ that showed strongest sensitivity to our contrast of interest. Another approach would be to apply a fixed functional ROI based on the group average. In both cases, individual outliers can be mitigated using a random effects procedure during model comparison (as we have done here). However, it remains unknown exactly how sensitive DCM analysis is to these differences in procedure. This issue is certainly not unique to our study. We are not able to test this rigorously in our own data as we do not have ground truth, i.e., we could apply a fixed ROI based on the group contrast, but as we have not generated the data that would go into each analysis, we do not exactly know how much the results of DCM analysis should differ between these analysis choices, nor exactly what these differences would mean with regards to what is the "correct" model to explain the data. Once again, a principled investigation using simulated data, where the ground truth is known, is required to meaningfully address these questions but is beyond the scope of the current work.

\section{Conclusions}

Here, we asked whether multitasking limitations are better associated with activity changes in a frontal-parietal, or a striatal-cortical network. Using DCM, we show evidence for the latter. Specifically, multitasking demands were associated with increased rates of coupling between the putamen and cortical sites. We interpret this as suggesting that performance decrements are due, at least in part, to a limit in the rate at which the putamen can excite appropriate cortical stimulus-response representations. Moreover, the observation that coupling strength between putamen and pre-SMA is modulated with practice and that the extent of the modulation differs between the practice and control groups suggests that multitasking limits may be remediated by changes in the rate of information transfer between the putamen and the pre-SMA, which can be observed early in practice. We also suggest that modulated rates of corticostriatal information transfer gained from practice over multiple days may be a key mechanism for supporting action representations under conditions of high cognitive load. These results provide clear empirical evidence that multitasking operations may not just be mediated by a frontal-parietal network. Rather, the interface between the putamen and key cortical nodes appear to correspond to multitasking operations, and the modulation of multitasking limitations.

\section{References}

Alexander GE, DeLong MR, Strick PL (1986) Parallel organization of functionally segregated circuits linking basal ganglia and cortex. Annu Rev Neurosci 9:357-381.
Allen M, Poggiali D, Whitaker K, Marshall TR, Kievit RA (2018) Raincloud plots: a multi-platform tool for robust data visualization. e27137v1. Wellcome Open Res 4:63.

Alloway KD, Smith JB, Mowery TM, Watson GDR (2017) Sensory processing in the dorsolateral striatum: the contribution of thalamostriatal pathways. Front Syst Neurosci 11:53.

Anderson JS, Ferguson MA, Lopez-Larson M, Yurgelun-Todd D (2010) Topographic maps of multisensory attention. Proc Natl Acad Sci USA 107:20110-20114.

Arnell KM, Duncan J (2002) Separate and shared sources of dualtask cost in stimulus identification and response selection. Cogn Psychol 44:105-147.

Ashby FG, Turner BO, Horvitz JC (2010) Cortical and basal ganglia contributions to habit learning and automaticity. Trends Cogn Sci 14:208-215.

Badre D, Nee DE (2018) Frontal cortex and the hierarchical control of behavior. Trends Cogn Sci 22:170-188.

Barnes TD, Kubota Y, Hu D, Jin DZ, Graybiel AM (2005) Activity of striatal neurons reflects dynamic encoding and recoding of procedural memories. Nature 437:1158-1161.

Boot WR, Simons DJ, Stothart C, Stutts C (2013) The pervasive problem with placebos in psychology: why active control groups are not sufficient to rule out placebo effects. Perspect Psychol Sci 8:445-454.

Bornstein AM, Daw ND (2011) Multiplicity of control in the basal ganglia: computational roles of striatal subregions. Curr Opin Neurobiol 21:374-380.

Borst JP, Taatgen NA, Stocco A, van Rijn H (2010) The neural correlates of problem states: testing FMRI predictions of a computational model of multitasking. PLoS One 5:e12966.

Bunge SA, Hazeltine E, Scanlon MD, Rosen AC, Gabrieli JDE (2002) Dissociable contributions of prefrontal and parietal cortices to response selection. Neuroimage 17:1562-1571.

Caballero JA, Humphries MD, Gurney KN (2018) A probabilistic, distributed, recursive mechanism for decision-making in the brain. PLoS Comput Biol 14:e1006033.

Camilleri JA, Müller VI, Fox P, Laird AR, Hoffstaedter F, Kalenscher T, Eickhoff SB (2018) Definition and characterization of an extended multiple-demand network. Neuroimage 165:138-147.

Cavada C, Goldman-Rakic PS (1989) Posterior parietal cortex in rhesus monkey: II. Evidence for segregated corticocortical networks linking sensory and limbic areas with the frontal lobe. J Comp Neurol 287:422-445.

Chein JM, Schneider W (2012) The brain's learning and control architecture. Curr Dir Psychol Sci 21:78-84.

Cole MW, Reynolds JR, Power JD, Repovs G, Anticevic A, Braver TS (2013) Multi-task connectivity reveals flexible hubs for adaptive task control. Nat Neurosci 16:1348-1355.

Crittenden BM, Duncan J (2014) Task difficulty manipulation reveals multiple demand activity but no frontal lobe hierarchy. Cereb Cortex 24:532-540.

David O, Guillemain I, Saillet S, Reyt S, Deransart C, Segebarth C, Depaulis A (2008) Identifying neural drivers with functional MRI: an electrophysiological validation. PLoS Biol 6:2683-2697.

Duncan J (2010) The multiple-demand (MD) system of the primate brain: mental programs for intelligent behaviour. Trends Cogn Sci 14:172-179.

Duncan J (2013) The structure of cognition: attentional episodes in mind and brain. Neuron 80:35-50.

Dux PE, Ivanoff J, Asplund CL, Marois R (2006) Isolation of a central bottleneck of information processing with time-resolved FMRI. Neuron 52:1109-1120.

Dux PE, Tombu MN, Harrison S, Rogers BP, Tong F, Marois R (2009) Training improves multitasking performance by increasing the speed of information processing in human prefrontal cortex. Neuron 63:127-138.

Erickson KI, Colcombe SJ, Wadhwa R, Bherer L, Peterson MS, Scalf PE, Kramer AF (2005) Neural correlates of dual-task performance after minimizing task-preparation. Neuroimage 28:967-979. 
Erickson KI, Colcombe SJ, Wadhwa R, Bherer L, Peterson MS, Scalf PE, Kim JS, Alvarado M, Kramer AF (2007) Training-induced functional activation changes in dual-task processing: an FMRI study. Cereb Cortex 17:192-204.

Filmer HL, Mattingley JB, Marois R, Dux PE (2013) Disrupting prefrontal cortex prevents performance gains from sensory-motor training. J Neurosci 33:18654-18660.

Friston KJ, Harrison L, Penny W (2003) Dynamic causal modelling. Neuroimage 19:1273-1302.

Friston KJ, Kahan J, Biswal B, Razi A (2014) A DCM for resting state fMRI. Neuroimage 94:396-407.

Garner KG, Dux PE (2015) Training conquers multitasking costs by dividing task representations in the frontoparietal-subcortical system. Proc Natl Acad Sci USA 112:14372-14377.

Garner KG, Tombu MN, Dux PE (2014) The influence of training on the attentional blink and psychological refractory period. Atten Percept Psychophys 76:979-999.

Garner KG, Matthews N, Remington RW, Dux PE (2015) Transferability of training benefits differs across neural events: evidence from ERPs. J Cogn Neurosci 27:2079-2094.

Garner KG, Lynch CR, Dux PE (2016) Transfer of training benefits requires rules we cannot see (or hear). J Exp Psychol 42:1148-1157.

Goard MJ, Pho GN, Woodson J, Sur M (2016) Distinct roles of visual, parietal, and frontal motor cortices in memory-guided sensorimotor decisions. Elife 5:e13764.

Graybiel AM, Grafton ST (2015) The striatum: where skills and habits meet. Cold Spring Harb Perspect Biol 7:a021691.

Grefkes C, Fink GR (2005) The functional organization of the intraparietal sulcus in humans and monkeys. J Anat 207:3-17.

Guo L, Walker WI, Ponvert ND, Penix PL, Jaramillo S (2018) Stable representation of sounds in the posterior striatum during flexible auditory decisions. Nat Commun 9:1534.

Haber SN (2016) Corticostriatal circuitry. Dialogues Clin Neurosci 18:7-21.

Hélie S, Ell SW, Ashby FG (2015) Learning robust cortico-cortical associations with the basal ganglia: an integrative review. Cortex 64:123-135.

Hesselmann G, Flandin G, Dehaene S (2011) Probing the cortical network underlying the psychological refractory period: a combined EEG-fMRI study. Neuroimage 56:1608-1621.

Jahanshahi M, Obeso I, Rothwell JC, Obeso JA (2015) A fronto-striato-subthalamic-pallidal network for goal-directed and habitual inhibition. Nat Rev Neurosci 16:719-732.

Jiang Y (2004) Resolving dual-task interference: an fMRI study. Neuroimage 22:748-754.

Jiang Y, Saxe R, Kanwisher N (2004) Functional magnetic resonance imaging provides new constraints on theories of the psychological refractory period. Psychol Sci 15:390-396.

Jin X, Costa RM (2010) Start/stop signals emerge in nigrostriatal circuits during sequence learning. Nature 466:457-462.

Joel D, Niv Y, Ruppin E (2002) Actor-critic models of the basal ganglia: new anatomical and computational perspectives. Neural Netw 15:535-547.

Jog MS, Kubota Y, Connolly CI, Hillegaart V, Graybiel AM (1999) Building neural representations of habits. Science 286:1745-1749.

Jolicoeur P (1998) Modulation of the attentional blink by on-line response selection: evidence from speeded and unspeeded task1 decisions. Mem Cognit 26:1014-1032.

Kelly AMC, Garavan H (2005) Human functional neuroimaging of brain changes associated with practice. Cereb Cortex 15:10891102.

Kim JH, Lee JM, Jo HJ, Kim SH, Lee JH, Kim ST, Seo SW, Cox RW, Na DL, Kim SI, Saad ZS (2010) Defining functional SMA and preSMA subregions in human MFC using resting state $\mathrm{fMRI}$ : functional connectivity-based parcellation method. Neuroimage 49:23752386.

Lever J, Krzywinski M, Altman N (2016) Model selection and overfitting. Nat Methods 13:703-704.
Luppino G, Matelli M, Camarda R, Rizzolatti G (1993) Corticocortical connections of area F3 (SMA-proper) and area F6 (pre-SMA) in the macaque monkey. J Comp Neurol 338:114-140.

Marois $\mathrm{R}$, Ivanoff $\mathrm{J}$ (2005) Capacity limits of information processing in the brain. Trends Cogn Sci 9:296-305.

Marois R, Larson JM, Chun MM, Shima D (2006) Response-specific sources of dual-task interference in human pre-motor cortex. Psychol Res 70:436-447.

Marti S, King JR, Dehaene S (2015) Time-resolved decoding of two processing chains during dual-task interference. Neuron 88:12971307.

Nachev P, Wydell H, O'neill K, Husain M, Kennard C (2007) The role of the pre-supplementary motor area in the control of action. Neuroimage 36 [Suppl 2]:T155-T163.

Nijboer M, Borst J, van Rijn H, Taatgen N (2014) Single-task fMRI overlap predicts concurrent multitasking interference. Neuroimage 100:60-74.

Penny WD, Stephan KE, Daunizeau J, Rosa MJ, Friston KJ, Schofield TM, Leff AP (2010) Comparing families of dynamic causal models. PLoS Comput Biol 6:e1000709.

Petersen SE, van Mier H, Fiez JA, Raichle ME (1998) The effects of practice on the functional anatomy of task performance. Proc Natl Acad Sci USA 95:853-860.

Pho GN, Goard MJ, Woodson J, Crawford B, Sur M (2018) Task-dependent representations of stimulus and choice in mouse parietal cortex. Nat Commun 9:2596.

Reig R, Silberberg G (2014) Multisensory integration in the mouse striatum. Neuron 83:1200-1212.

Ruthruff E, Johnston JC, Van Selst M (2001) Why practice reduces dual-task interference. J Exp Psychol Hum Percept Perform 27:321.

Saint-Cyr JA, Ungerleider LG, Desimone R (1990) Organization of visual cortical inputs to the striatum and subsequent outputs to the pallido-nigral complex in the monkey. J Comp Neurol 298:129-156.

Sigman M, Dehaene S (2008) Brain mechanisms of serial and parallel processing during dual-task performance. J Neurosci 28:75857598.

Slagter HA, Mazaheri A, Reteig LC, Smolders R, Figee M, Mantione M, Schuurman PR, Denys D (2017) Contributions of the ventral striatum to conscious perception: an intracranial EEG study of the attentional blink. J Neurosci 37:1081-1089.

Smith KS, Graybiel AM (2013) A dual operator view of habitual behavior reflecting cortical and striatal dynamics. Neuron 79:361374.

Stelzel C, Schumacher EH, Schubert T, D'Esposito M (2006) The neural effect of stimulus-response modality compatibility on dualtask performance: an fMRI study. Psychol Res 70:514-525.

Stephan KE, Penny WD, Daunizeau J, Moran RJ, Friston KJ (2009) Bayesian model selection for group studies. Neuroimage 46:10041017.

Strobach T, Torsten S (2017) Mechanisms of practice-related reductions of dual-task interference with simple tasks: data and theory. Adv Cogn Psychol 13:28-41.

Szameitat AJ, Schubert T, Müller K, Von Cramon DY (2002) Localization of executive functions in dual-task performance with fMRI. J Cogn Neurosci 14:1184-1199.

Szameitat AJ, Lepsien J, von Cramon DY, Sterr A, Schubert T (2006) Task-order coordination in dual-task performance and the lateral prefrontal cortex: an event-related fMRI study. Psychol Res 70:541-552.

Telford CW (1931) The refractory phase of voluntary and associative responses. J Exp Psychol 14:1-36.

Thoma P, Koch B, Heyder K, Schwarz M, Daum I (2008) Subcortical contributions to multitasking and response inhibition. Behav Brain Res 194:214-222.

Thorn CA, Atallah H, Howe M, Graybiel AM (2010) Differential dynamics of activity changes in dorsolateral and dorsomedial striatal loops during learning. Neuron 66:781-795. 
Tombu MN, Asplund CL, Dux PE, Godwin D, Martin JW, Marois R (2011) A Unified attentional bottleneck in the human brain. Proc Natl Acad Sci USA 108:13426-13431.

Verghese A, Garner KG, Mattingley JB, Dux PE (2016) Prefrontal cortex structure predicts training-induced improvements in multitasking performance. J Neurosci 36:2638-2645.

Verghese A, Mattingley JB, Garner KG, Dux PE (2018) Decision-making training reduces the attentional blink. J Exp Psychol 44:195205.

Vossel S, Weidner R, Driver J, Friston KJ, Fink GR (2012) Deconstructing the architecture of dorsal and ventral attention systems with dynamic causal modeling. J Neurosci 32:1063710648.

Vossel S, Geng JJ, Fink GR (2014) Dorsal and ventral attention systems: distinct neural circuits but collaborative roles. Neuroscientist 20:150-159.

Watanabe K, Funahashi S (2014) Neural mechanisms of dual-task interference and cognitive capacity limitation in the prefrontal cortex. Nat Neurosci 17:601-611.

Watanabe K, Funahashi S (2018) Toward an understanding of the neural mechanisms underlying dual-task performance: contribution of comparative approaches using animal models. Neurosci Biobehav Rev 84:12-28.

Wise SP, Boussaoud D, Johnson PB, Caminiti R (1997) Premotor and parietal cortex: corticocortical connectivity and combinatorial computations. Annu Rev Neurosci 20:25-42.

Woolgar A, Hampshire A, Thompson R, Duncan J (2011) Adaptive coding of task-relevant information in human frontoparietal cortex. J Neurosci 31:14592-14599.

Yartsev MM, Hanks TD, Yoon AM, Brody CD (2018) Causal contribution and dynamical encoding in the striatum during evidence accumulation. Elife $7:$ e34929.

Yildiz A, Beste C (2015) Parallel and serial processing in dual-tasking differentially involves mechanisms in the striatum and the lateral prefrontal cortex. Brain Struct Funct 220:3131-3142.

Yin HH, Knowlton BJ (2006) The role of the basal ganglia in habit formation. Nat Rev Neurosci 7:464-476.

Zylberberg A, Fernández Slezak D, Roelfsema PR, Dehaene S, Sigman M (2010) The brain's router: a cortical network model of serial processing in the primate brain. PLoS Comput Biol 6: e1000765. 